\title{
ACGA Algorithm of Solving Weapon - Target Assignment Problem
}

\author{
Jiuyong Zhang ${ }^{1,2}$ \\ ${ }^{1}$ Mathematics Department, \\ Academy of Armed Forces Engineering, \\ Beijing, 100072, China \\ ${ }^{2}$ School of Science, Beijing University of Civil Engineering \\ and Architecture, Beijing, 100044 , China \\ Chuanqing $\mathrm{Xu}^{2,3}$ \\ ${ }^{2}$ School of Science, Beijing University of Civil Engineering \\ and Architecture, Beijing, 100044 , China \\ ${ }^{3}$ Mathematics Department, Hanshan Normal College \\ Chaozhou, 521041, China
}

\author{
Xiaojing Wang ${ }^{2}$ \\ ${ }^{2}$ School of Science, \\ Beijing University of Civil Engineering and Architecture, \\ Beijing, 100044 , China \\ Dehui Yuan ${ }^{3}$ \\ ${ }^{3}$ Mathematics Department, \\ Hanshan Normal College \\ Chaozhou, 521041, China
}

\begin{abstract}
Weapon Target Assignment is not only an important issue to use firepower, but also an important operational decision-making problem. As new intelligent algorithms, Genetic algorithm and ant colony algorithm are applied to solve Weapons-Target Assignment Problem. This paper introduces the Weapon-Target Assignment (WTA) and the mathematical model, and proposes ACGA algorithm which is the integration of genetic algorithm and ant colony algorithm then use ACGA algorithm to solve the Weapon-Target Assignment Problem. Calculations show that: when ACGA algorithm is used to solve Weapon - Target Assignment Problem, it has fast convergence and high accuracy.
\end{abstract}

Keywords- Weapon -Target Assignment; ant colony algorithm; genetic algorithm; integration

\section{Introduction}

In the modern military war, weapons-target assignment is a very important problem. The timeliness of weaponstarget assignment and the quality of scheme directly influence the effect of the combat. Therefore, people have made a lot of algorithms to solve this problem: Firstly, traditional algorithms, including implicit enumeration method, the branch and bound method, cutting plane method, the dynamic programming, etc; Secondly, intelligent algorithms, such as simulated annealing algorithm, neural network algorithm, genetic algorithm, ant colony algorithm, etc; Thirdly, the algorithm which is combined with two kinds of algorithms is used to solve the problem [1].

\section{Basic Concept and Mathematical Model of WTA}

The definition of WTA: according to the enemy's ruing probability, our defensive weapons' kill probability and the important degree of the resources, by some allocation strategies, before the enemy's attacks, scientific distribute our defensive weapons, to make the best hitting of enemy or the best protection of our defense resources. If targets distributed to weapons is one-time, this is called static distribution. In actual combat, the situation is in the twinkling of an eye, the actual results of the war and the expected effect can not perfectly the same, in order to further improve the defense quality, according to the real-time situation of the war, it is necessary to more stages to distribute weapons, this is called dynamic allocation.

Weapons-target distribution (WTA) described as: In a battle, there are $\mathrm{m}$ kinds of weapon platforms $W_{1}, W_{2}, \cdots, W_{m}$, existing $\mathrm{n}$ targets $T_{1}, T_{2}, \cdots, T_{n}$, the value of targets is $V_{1}, V_{2}, \cdots, V_{n}$, the weapon platform $i$ ( $i=1,2, \cdots, m)$ has weapons $r_{i}$, Target $T_{j}$ is distributed weapons $S_{j}$ at most, the probability of weapon platforms $W_{i}$ distribute to target $T_{i}$ is $q_{i j}$ $(i=1,2, \cdots, m, j=1,2, \cdots, n)$.

Different combat purposes have different mathematical models. There are kinds of standards as following: (1) the maximum probability or expect of defenders' survival; (2) the minimum probability or expect of enemy survival; (3) the weighted sum of the first standard and the second standard, namely on the one hand that to the maximum probability or expect of defenders' survival, on the other hand to the minimum probability or expect of enemy survival, the mathematical model of this standards is complex.

The optimal allocation goal of this paper is the minimum failure value of weapons hit all of the targets. 
Suppose that $x_{i j}$ is the number of weapons platform $i$ attacks target $j$.the WTA model is:

$$
\begin{aligned}
& \min E=\sum_{j=1}^{n} V_{j} \prod_{i=1}^{m}\left(1-q_{i j}\right)^{x_{i j}} \\
& \left\{\begin{array}{l}
\sum_{i=1}^{m} x_{i j} \leq s_{j}, j=1,2, \cdots n \\
\sum_{j=1}^{n} x_{i j} \leq r_{i}, i=1,2, \cdots m
\end{array}\right.
\end{aligned}
$$

WTA is NP problem in essence [2], there is no effective algorithm for solving this problem.

\section{Integration of ant colony algorithm and genetic algorithm}

\section{A. Ant colony algorithm theory}

Ant Colony Algorithm is presented by Italian scholars such as Colorni[3]-[5], in the early $1990 \mathrm{~s}$ they simulated the behavior of collective ants foraging in nature and proposed the heuristic optimization algorithm based on the bionic population. Firstly they used ACO success in solving the famous Traveling salesman Problem (TSP). Below we use TSP as an example to explain to the ant colony algorithm. Suppose that $\mathrm{C}$ is the set of the cities which number is $\mathrm{n}$, and the ant number is $\mathrm{m}$, not $d_{i j}(i, j=1,2, \cdots, n)$ is the distance between city $\mathrm{i}$ and city $\mathrm{j} . \tau_{i j}(t)$ is the residual pheromone strength of the path which is between city $i$ and city $\mathrm{j}$ at time $\mathrm{t}$, in order to simulate the real secretion of the ants. In athletic process, the ant $\mathrm{k}(\mathrm{k}=1,2, \cdots, \mathrm{m})$ determine its transfer direction according to the amount of information on various paths, and we use the taboo watch $\operatorname{tabu}_{k}(k=1,2, \cdots, n)$ to record the city which is the ant $\mathrm{k}$ had passed through. In the search process, according to the information and inspiration information of various paths, the ant calculates the transition probability, so as to determine the next city. $p_{i j}^{k}(t)$ means the probability of the ant $\mathrm{k}$ from city $i$ transfer to city $j$ at time $t$, and its expression is:

$$
p_{i j}^{k}(t)= \begin{cases}\frac{\left[\tau_{i j}(t)\right]^{\alpha} \cdot\left[\eta_{i k}(t)\right]^{\beta}}{\sum_{s \in \text { allowed }}\left[\tau_{i j}(t)\right]^{\alpha} \cdot\left[\eta_{i k}(t)\right]^{\beta}}, & \text { if } \\ 0 & \text {, else }\end{cases}
$$

In formula (2):

allowed $_{k}=\left\{C-\right.$ tabu $\left._{k}\right\}$ means the next city which is allowed ant k to choose; $\alpha$ is the information heuristic factor, means the relative importance of track, it reflects the role of information which is ants accumulate in athletic process. The bigger the value, the more the ant inclines to choose the path which is other ants had passed through, and the more coordination between the ants; $\beta$ is the expect stimulating factor, means the relative importance of visibility, it reflects the importance of the elicitation information in athletic process, the bigger the value, the closer the transition probability to greed rules; $\eta_{i j}(t)$ is inspired function, its expression as follows:

$$
\eta_{i j}(t)=\frac{1}{d_{i j}}
$$

In order to avoid too much residue information to submerge residual heuristic information, when an ant walks one step, or completes all of the cities (also known as a loop, we must update residue information. This update imitate the characteristics of human brain memory, when new information into the brain continuously, the old information stored in the brain with the passage of time decline, or even forget. Therefore, the information in the path $(i, j)$ at time $\mathrm{t}+1$, is adjusted following rules:

$$
\begin{array}{r}
\tau_{i j}(t+1)=(1-\rho) \cdot \tau_{i j}(t)+\Delta \tau_{i j}(t) \\
\Delta \tau_{i j}(t)=\sum_{k=1}^{m} \Delta \tau_{i j}^{k}(t)
\end{array}
$$

In formula (4) and (5), $\rho$ means pheromone volatile coefficient, so $1-\rho$ notes pheromone residual factor. In order to prevent the infinite accumulation of information, the values of $\rho$ for $\rho \subset[0,1) ; \Delta \tau_{i j}(t)$ means the pheromone increment at path $(\mathrm{i}, \mathrm{j})$ in the cycle and $\Delta \tau_{i j}(0)=0, \Delta \tau_{i j}^{k}(t)$ means the information of ant $\mathrm{k}$ residue sin the path $(i, j)$ in the cycle.

In the Ant-Cycle which is the most commonly used model,

$$
\Delta \tau_{i j}(t)=\left\{\begin{array}{l}
\frac{Q}{L_{k}},(i, j) \in L_{k} \\
\mathrm{o}, \quad \text { else }
\end{array}\right.
$$

In formula (6), $\mathrm{Q}$ is pheromone strength which is constant. It influences the algorithm convergence speed to a certain extent. $\mathrm{L}_{k}$ means the total length of the paths which is the ant $\mathrm{k}$ walked in the cycle. When all the ants travel all the cities, we should global update the residual information. And we only update the optimal path $\mathrm{L}$ in this cycle, for its expression:

$$
\Delta \tau_{i j}(t)=\left\{\begin{array}{l}
\frac{Q}{L},(i, j) \in L(7) \\
0, \text { else }
\end{array}\right.
$$

\section{B. Integration of ant colony algorithm and genetic algorithm}

Compared with intelligent algorithms such as the genetic algorithm, the chaos algorithm, particle swarm algorithm, the main advantages of ant colony algorithm is that: (1) Strong robustness. To modify slightly ant colony algorithm model, it can be applied to other problems; (2) Distributed computing; (3) Combined with other methods easily. Of course, it also has some defects: The algorithm is generally need longer search time, and is prone to stagnation phenomenon, when groups is in large scale, it is difficult to find out a best path in a short time.

Genetic algorithm which is proposed by American professor JohnHollnad in 1975, is a type of bionic optimization algorithm. It uses population search technology, gives a series of genetic operations on the current population such as selection, crossover, mutation and so on. Then produces a new generation of population and 
gradually make the evolution close to the optimal solution. It has rapid global search capability in a wide range. But when it reaches a certain range, it will make a lot of redundancy iteration. In a word, it has low efficiency for the exact solution.

The idea of ant colony algorithm integrates with genetic algorithm is: Firstly, we produce $m$ optimal paths[7], the paths stay information, others is unchanged. Then use ant colony algorithm to complete the cycles, record the optimal and near-optimal solution each cycle. For the cycle we give crossover and mutation on optimal and near-optimal solution to reach the new optimal solution. When we complete all the cycles, we also get the global optimal solution.

\section{The principle of ACGA algorithm and the solution steps of the WTA}

The ant colony algorithm had been used to solve WTA problem[6]. The ACGA algorithm, which is proposed this paper, is improved on the basis of the ant colony algorithm. It brings random number and pheromone updating strategy to expand the diversity of the initial solution. And brings crossover operator and mutation operator to enhance the ability of looking for the global optimal solution and accelerate the convergence speed.

This paper utilizes real number code, the length of code is the number of targets, take 10 weapons hit 10 targets for example: [1-4-5-6-2-3-9-10-8-7] means weapon 1 hit target 1 , weapon 2 hit target 5 , weapon 3 hit target 6 , weapon 4 hit target 2 , weapon 5 hit target 3 , weapon 6 hit target 4 , weapon 7 hit target 10, weapon 8 hit target 9 , weapon 9 hit target 7, weapon 10 hit target 8 .

\section{Random number}

When we calculate the transition probability $p_{i j}^{k}(t)$, we bring the random number $0.1+0.8 *$ rand (1),

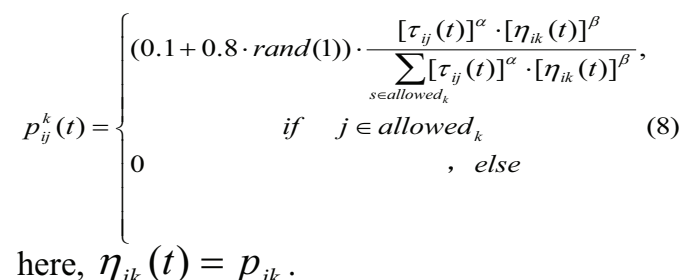

So it increases the random selection and the diversity of the solution, to avoid the algorithm reach the local optimal early. And the algorithm is simple that can't increase the complexity of the algorithm.

\section{Pheromone updating strategy}

We use the pheromone diminishing strategy which is mentioned in references[8][9] for the local pheromones updating strategy.

$$
\Delta \tau_{i j}^{k}=\left\{\begin{array}{l}
q_{i j} \frac{\operatorname{Max}-\frac{(n-i+1)(\operatorname{Max}-\operatorname{Min})}{n}}{(n-1)(\operatorname{Max}+\min ) / 2}, \text { if }(i, j) \subset L \\
0 \quad \text { else }
\end{array}\right.
$$

In it, Max means the maximum updating constant of the pheromone; Min means the minimum updating constant of the pheromone.

And the global pheromone updating strategy is

$\tau_{i j}(t+1)=(1-\rho) \cdot \tau_{i j}(t)+q_{i j} \cdot \Delta \tau_{i j} / \min E$

As the new updating strategy, it can avoid the information in the local optimal path much higher than others. It also unlimited the further search space that the algorithm isn't prone to premature, stagnation phenomenon.

\section{E. Crossover operator}

We select randomly the same part of the optimal solution $C_{1}$ and near-optimal solution $C_{2}$ to crossover, and produce two new solutions $N e w_{1}$ and $N e w_{2}$, then we get the optimal solution $\mathrm{New}$ from $\mathrm{C}_{1}, \mathrm{New}$ and $\mathrm{New} \mathrm{w}_{2}$. For example, suppose that the optimal solution $C_{1}=[1-2-3-4-5-$ 6-9-8-7] and near-optimal solution $C_{2}=[1-2-3-6-5-4-7-8-9]$, the crossover part is from 4 to 6 , that's means we should crossover the [4-5-6] in $C_{1}$ with the [6-5-4] in $C_{2}$. We can get $N e w_{1}=[1-2-3-6-5-4-9-8-7], \mathrm{New}_{2}=[1-2-3-4-5-6-7-8$ 9]. Then according to the values of $\mathrm{E}$, we can get the optimal solution $\mathrm{New}$ from $\mathrm{C}_{1}, \mathrm{New}$ and $\mathrm{New}$.

\section{F. mutation operator}

If the solution New meets the mutation probability, to get the mutated solution $M u$, we should select randomly the mutation position and change it with the one in front of it. Then we get the local optimal solution from New and $M u$. For example, New = [1-2-3-6-5-4-9-8-7], the mutation position is 3, then the mutated solution $\mathrm{Mu}=[1-3-2-6-5-4-9$ $8-7]$. According to the values of $\mathrm{E}$, we can get the local optimal solution.

\section{G. Steps of ACGA algorithm}

The steps of ACGA algorithm to solve WTA are:

Step 1: Initialize parameters.

Step 2: Use genetic algorithm to produce solutions, leave pheromone on the paths of the solutions, other paths unchanged.

Step 3: Cycles $n c \leftarrow n c+1$.

Step 4: $\mathrm{m}$ ants are placed in the weapon platform i, according to the probability $p_{i j}^{k}$, the ant $\mathrm{k}$ transfer to target $\mathrm{j}$, until completes the traversal. In the traversal process, according to the constraints of weapons and targets, we should update the weapons and targets taboo list.

Step 5: According to formula (9), update the local pheromone in the process.

Step 6 : From the $\mathrm{m}$ traversals, get the solution $C_{1}$ and near-optimal solution $C_{2}$. 
Step 7 : Crossover $C_{1}$ with $C_{2}$ to get $N e w_{1}$ and $N e w_{2}$, get optimal solution New.

Step 8: According to the mutation probability $p_{m}=0.01$, mutate the solution New to get the solution Mu. Compared with New and Mu, we get the local optimal solution.

Step 9: According to formula (10), update the global pheromone in the local optimal solution.

Step 10: If the number of cycles nc $\geqslant N \max$, end the cycle and output the results, otherwise empty the tabu list and jump to step 2 .

\section{Algorithm testing and analysis}

In order to verify the validity of the ACGA algorithm, take $\mathrm{m}=14, \mathrm{n}=14$ for example, the experimental data from reference [10].

We use ant colony algorithm, genetic algorithm and SAGA algorithm to simulate. Each algorithm runs 100 times, we select 20 of the best solution as samples. The basic settings are as follows:

$$
\begin{aligned}
& \alpha=1, \beta=5, \rho=0.7, Q=150, N \max =500, \\
& \operatorname{Max}=0.96, \text { Min }=0.05
\end{aligned}
$$

Table 1 gives the optimal $\mathrm{E}(\mathrm{oE})$, average $\mathrm{E}(\mathrm{aE})$, maximum times(maT), minimum times(miT), average times(aT) when use the three algorithms to solve WTA problem.

TABLE I. RESULTS OF THREE ALGORITHMS

\begin{tabular}{|c|c|c|c|c|c|}
\hline & $\mathbf{o E}$ & $\mathbf{a E}$ & $\mathbf{m a T}$ & $\mathbf{m i T}$ & $\mathbf{a T}$ \\
\hline $\mathbf{A C}$ & 4.16 & 4.92 & 444 & 2 & 179.6 \\
\hline GA & 4.39 & 4.69 & 478 & 9 & 223.8 \\
\hline ACGA & 2.68 & 3.13 & 307 & 9 & 224.3 \\
\hline
\end{tabular}

From the table we can see that, the results of ASGA algorithm is better than ant colony algorithm and genetic algorithm, that's means ASGA is superior to the other algorithms. From the iterative times, the times of ant colony algorithm are fewer than the other algorithms. That means ant colony algorithm has convergence speed, but the accuracy is not very high. In a word, the results show that ASGA algorithm is superior to the other algorithms.

\section{Conclusion}

To solve WTA problem especially large-scale has important theoretical significance and practical value. As intelligent algorithms, ant colony algorithm and genetic algorithm are applied to solve WTA problem. They have different advantages, of course, also have shortcomings. This paper has studied the integration of genetic algorithms and ant colony algorithm, bring random number and pheromone updating strategy to increase the diversity of the solutions; bring crossover operator and mutation operator to expand the search space. The improved algorithm of ACGA has strong global search ability and fast convergence speed. The simulated example shows that this article is an effective improved algorithm.

\section{Acknowledgment}

We would like to thank all the researchers who provided their assistance. We are grateful to Professor Songgui Wang for constructive criticisms on the manuscript. * Corresponding author: yaoxcq@126.com,13810720446.Supported by NSFC with No.30800244, 60971075 and by the Natural Science Foundation of Guangdong Province with No.7008110

\section{REFERENCES}

[1] CAI Huai-ping,CHEN Ying-wu. The Development of the Research on Weapon-Target Assignment (WTA) problem $[\mathrm{J}]$.Fire Control and Command Control,2006,31(12):13-15

[2] S.P.Lloyd,H.S.Witsenhause. Weapon allocation is NPComplete. Proceeding of the IEEE Summer Simulation Conference. Reno, Nevada, 1986:1054 1058

[3] Colorni A,Dorigo M,Maniezzo V,et al.Distributed optimization by ant colonies [A]. Proceedings of European Conference onArtificialLife.Paris, 1991: 134142.

[4] Colorni A,Dorigo M,M aniezzo V.An investigation of some properties of an ant algorithm.Proceedings of Parallel Problem Solving from Nature(PPSN). France: Elsevier, 1992:509-520.

[5] Dorigo M,Maniezzo V,Colorni A.Ant system: Optimization by a colony cooperating Agents[J].IEEE Trans.on Systems,Man andCybernetics.Part B:Cybernetics(S1083-4419),1996,26(1):29-41.

[6] GAO Shang,Ant Colony Algorithm for Weapon-target Assignment problem[J]. Computer Engineering and Applications, 2003,78-79

[7] DING Jian-Li, CHEN Zeng-Qiang, and YUAN ZhuZhi, On the Combination of Genetic Algorithm and Ant Algorithm[J], Journal of Computer Research and development, 2003,40(9): 1351-1354

[8] MA Xi-Jun,PAN Ruo-Yu,YANG Shan-Lin, Ant Colony Algorithm Based on Pheromone Declining[J] Journal of System Simulation,2006,18(11):3297-3300.

[9] YUANMei, LINGMing-xiang, ZENG Qing-shuang, An AntColony Algorithm Based on Pheromone Declining for Solving theWTA Problem[J], Computer Simulation,2008,25(2);23-25

[10] CAO Qiying,HE Zhangbing.A Genetic Algorithm of Solving WTA Problem[J].Control Theory and Applications , 2001, 18(1): 76-79. 